\title{
Coordinated Voltage Control in Distribution Network with the Presence of DGs and Variable Loads Using Pareto and Fuzzy Logic
}

\author{
José Raúl Castro ${ }^{1,2, *}$, Maarouf Saad ${ }^{3,+}$, Serge Lefebvre ${ }^{4,+}$, Dalal Asber ${ }^{4,+}$ and Laurent Lenoir ${ }^{4,+}$ \\ 1 Departamento de Ciencias de la Computación y Electrónica, Universidad Técnica Particular de Loja, \\ Loja 11-01-608, Ecuador \\ 2 Power Electronics and Industrial Control Research Group (GREPCI), Ecole de technologie supérieure, \\ Montréal, QC H3C 1K3, Canada \\ 3 Department of Electrical Engineering, Ecole de technologie supérieure, 1100, Notre-Dame Ouest, \\ Montréal, QC H3C 1K3, Canada; maarouf.saad@etsmtl.ca \\ 4 Hydro-Québec's research institute (IREQ), 1800, boul. Lionel-Boulet, Varennes, QC J3X 1S3, Canada; \\ lefebvre.serge@ireq.ca (S.L.); asber.dalal@ireq.ca (D.A.); lenoir.laurent@ireq.ca (L.L.) \\ * Correspondence: jrcastro@utpl.edu.ec; Tel.: +593-7370-1444 (ext. 2516); Fax: +593-7370-1444 \\ + These authors contributed equally to this work.
}

Academic Editor: Josep M. Guerrero

Received: 9 July 2015; Accepted: 13 January 2016; Published: 17 February 2016

\begin{abstract}
This paper presents an efficient algorithm to solve the multi-objective (MO) voltage control problem in distribution networks. The proposed algorithm minimizes the following three objectives: voltage variation on pilot buses, reactive power production ratio deviation, and generator voltage deviation. This work leverages two optimization techniques: fuzzy logic to find the optimum value of the reactive power of the distributed generation (DG) and Pareto optimization to find the optimal value of the pilot bus voltage so that this produces lower losses under the constraints that the voltage remains within established limits. Variable loads and DGs are taken into account in this paper. The algorithm is tested on an IEEE 13-node test feeder and the results show the effectiveness of the proposed model.
\end{abstract}

Keywords: coordinated voltage control; distributed generation; on load tap changer; multi-objective voltage control; fuzzy logic

\section{Introduction}

Due to rapid industrialization and growth of residential and commercial sectors, the electrical energy requirements have increased significantly over the last decades. In this situation, renewable energy becomes a very important factor in the electrical distribution system. This type of generating unit is known as distributed generation (DG), and these generators will supply a large portion of demand and many of them will be directly connected to the distribution network. The DGs may trigger variations in voltage and can cause a change of direction in the power flow. The voltage rise depends on the amount of energy injected by the DG and, therefore, it is a limiting factor for the DG capacity. Many researchers have studied DGs and their impact on the voltage, the reduction of the losses in the active and reactive power, and the maximization of the DG capacity [1-3]. In [4] a minimization of loss was used to determine the optimum size and location of DG.

On the other hand, a review of the literature shows that many works have been done assuming that the loads in the electrical network are fixed. There are only a few works that use variable loads [5-9]. In this paper, all the loads of the analyzed networks are varying in time to better reflect 
system operation. Three different models of load variation are utilized. Each model represents the measurements of the change in consumption of customers for $48 \mathrm{~h}$ (data provided by Hydro-Québec).

Coordinated voltage control (CVC) in distribution network adjusts the voltage in pilot buses. CVC uses the multi-objective problem to minimize the voltage variation at the pilot buses [10]. Several methods have been proposed to solve the optimization of the multi-objective (MO) voltage control problem. In [10] a genetic algorithm (GA) was used to determine an optimal weighted solution of the MO problem. In [11] a simpler evolution scheme for MO problems is proposed; this algorithm uses the local search for the generation of new candidate solutions.

Some researchers $[10,12,13]$ solve the MO voltage control problem converting the objectives into a single objective (SO) function; in this case, the objective is to find the solution that minimizes or maximizes this single objective. The optimization solution results in a single value that represents a compromise among all the objectives [13].

Other researchers [13-15] work with the objectives of the MO problem separately, resulting in a set of solutions called the Pareto frontier. This causes the difficulty to find an optimal solution since there is no a single solution. Therefore, a decision-maker (DM) is necessary to choose the most appropriate solution. This feature is useful because it provides a better understanding of the system because all the objectives are explored. This method leads to find the weighted minimum of the objectives. Thus, the constraints and criteria specified of each objective are important to find the Pareto frontier.

Electrical power systems are very difficult to control with traditional methods due to highly complex and nonlinear behaviors. Fuzzy logic can overcome these difficulties. In [16,17] a fuzzy logic technique was introduced to solve the optimal values of $\mathrm{MO}$ voltage control problem. The solution set is usually not a singleton set. The problem requires the objectives functions to be linear and it also requires the value of the minimal solutions of the system. To solve this problem, fuzzy logic can be used closely with other optimization technique [18].

Previous methods adequately solved the problem of MO voltage control problem using DGs in distribution networks obtaining optimum values of voltage and reactive power [3,4,10,16,19-23]. There is no research that calculates the value of the reactive power of the DG using the optimal values of the $\mathrm{MO}$ voltage control problem in distribution network with variable and unbalanced loads.

To overcome the problems cited above, this paper proposes a new method called coordinated voltage control using Pareto and fuzzy logic (CVCPF). This technique finds the optimal values of the MO voltage control problem and finds the optimal value of reactive power of the DG. CVCPF maintains the voltage of the buses into the established limits, minimize the losses of the network, and minimizes the voltage variation in the pilot bus. This new method is tested on an IEEE 13-node test feeder using variables and unbalanced loads.

CVCPF uses Pareto optimization for solving the MO voltage control problem; the objectives of the $\mathrm{MO}$ problem are resolved separately. This paper uses fuzzy logic to find the optimal reactive power of DG to inject in distribution system. Fuzzy logic analyzes the voltage difference $(\Delta V)$ between the reference voltage $\left(V_{\text {pref }}\right)$ and the optimal voltage of pilot bus $\left(V_{p O p t i m o}\right)$ to find the reactive power of DG that minimizes voltage error.

The original contributions of this paper consist basically in combining the following:

(1) Variables and unbalanced loads with DGs in distribution network are investigated.

(2) CVCPF uses two optimization techniques. Pareto Optimization to find the optimal voltage and fuzzy logic to calculate the optimal value of reactive power of DG.

(3) CVCPF uses the reactive power of DG as a control variable to minimize the voltage variation.

(4) The objectives of the MO voltage control problem are resolved separately.

The rest of this paper is organized as follows: Section 2 presents the classical CVC. Section 3 presents coordinated voltage control using Pareto and fuzzy approach (CVCPF). Simulation results are presented in Section 4 and, finally, in Section 5 the conclusions are given. 


\section{Coordinated Voltage Control (CVC)}

Richardot et al. in [10] demonstrated that CVC for transmission networks can be successfully applied to a distribution network. Based on this work, it is presented in the following subsections the optimization model considered in this paper.

\subsection{Objectives Function}

The voltage variation at the pilot buses, the reactive power production, and the generator's voltage deviations are coupled variables and are tied together. Any increase or decrease in voltage at pilot buses will increase or decrease the reactive power production and generator voltage respectively. These objectives are modelled as follows:

\subsubsection{Voltage at Pilot Bus}

The first objective is to minimize the variation in voltage at the pilot buses. In a mathematical form, the objective can be written as follows:

$$
F_{1}=\sum_{i \in P} \lambda_{i}\left[k\left(V_{i}^{r e f}-V_{i}\right)-\sum_{k \in G} C_{i, k}^{V} \cdot \Delta V_{k}\right]^{2}
$$

where:

$P$ and $G$ are the sets of pilot and generator buses indices; $V_{i}^{r e f}, V_{i}$ and $\Delta V_{k}$ are set-point voltage, actual voltage and voltage deviation at bus $i$, i.e., the difference of voltage values between two computing steps; $C_{i, k}^{V}$ is the sensitivity matrix coefficient linking the voltage variation at bus $i$ and bus $k$, respectively, $\lambda_{i}$ and $k$ are weighting factor and regulator gain, respectively.

\subsubsection{Reactive Power}

The second objective is the management of the reactive power. This objective is modelled as follows:

$$
F_{2}=\sum_{i \in G} \lambda_{i}^{q}\left[k\left(q^{r e f}-\frac{Q_{i}}{Q_{i}^{M A X}}\right)-\sum_{k \in G} C_{i, k}^{Q} \cdot \Delta V_{k}\right]^{2}
$$

where:

$G$ is the set of generator buses indices; $Q_{i}$ and $Q_{i}^{M A X}$ are actual and maximum reactive power generations at bus $i ; q^{r e f}=\sum_{i \in G} Q_{i} / \sum_{i \in G} Q_{i}^{M A X}$ is the uniform set-point reactive power value within the regulated area; $C_{i, k}^{Q}$ is sensitivity matrix coefficients linking, respectively, voltage variation at bus $i$ and bus k. $\lambda_{i}^{q}$ and $k$ are weighting factor and regulator gain, respectively.

\subsubsection{Voltage at Generators}

The third objective is the minimization of the generator's voltage deviations. The mathematical model is as follows:

$$
F_{3}=\sum_{i \in G} \lambda_{i}^{v}\left[k\left(V_{i}^{r e f}-V_{i}\right)-\Delta V_{i}\right]^{2}
$$

where:

$G$ is the set of generator buses indices; $V_{i}^{r e f}, V_{i}$ and $\Delta V_{i}$ are the set-point voltage, actual voltage and voltage deviation, respectively, at the bus $i$, i.e., the difference of voltage values between two computing steps. $\lambda_{i}^{v}$ and $k$ are weighting factor and regulator gain, respectively. 


\subsection{Constraints}

The constraints are presented as follows:

\subsubsection{Reactive Power Constraint}

In this work, one of the main objectives is to control the production of the reactive power of the DG. In [3] an acceptable power factor is of \pm 0.91 .

$$
q^{r e f}=\sum_{i \in G} Q_{i} / \sum_{i \in G} Q_{i}^{M A X}
$$

where: $\left|Q_{i}\right| \leqslant Q_{i}^{\max }$.

\subsubsection{Technical Compliance Voltage}

The compliance of constraints of voltage on the pilot and generator buses is used to determine the safe operation values. In distribution networks an acceptable steady voltage range is considered within $\pm 3 \%$ of the operating voltage at DG [24]:

$$
\begin{gathered}
V_{i} \in\left[V_{i}^{\text {min }} ; V_{i}^{M A X}\right] \text { for } i \in P \cup G \\
\left|\Delta V_{i}\right| \leqslant \Delta V_{i}^{M A X} \text { for } i \in G
\end{gathered}
$$

\subsubsection{Weights Constraints}

The weights of the objectives are important because they give priority to an objective that depends on the conditions of operation. For example, if the voltage on the pilot bus is outside of the limits, the weight for this objective will be higher than the other two; however, these weights are related as described in relation Equation (6):

$$
\lambda_{i}+\lambda_{i}^{q}+\lambda_{i}^{v}=1
$$

$\lambda_{i}, \lambda_{i}^{q}, \lambda_{i}^{v}$ are weighting factors for bus $i$.

\section{Coordinated Voltage Control Using Pareto and Fuzzy Logic (CVCPF)}

This section presents the Pareto optimization to find the optimal voltage on the pilot bus and the determination of reactive power of DG using a fuzzy approach.

\subsection{Pareto Optimization}

The classical methods consist of converting the MO problem into a single objective (SO) problem. The solution of this SO problem yield a single result that depend of the selection of the weights. On the other hand, Pareto optimization optimizes all objectives separately.

Figure 1 shows that Pareto optimization calculates a set of solutions called the Pareto frontier, which can optimize the maximum possible number of objectives. In this work, we use Matlab to find the minimum of multiple functions using a genetic algorithm and obtain the Pareto frontier subject to the linear equalities $A e q \times x=b e q$. All objectives and constraints are changing in the real-time set considering the actual needs and capabilities. This Pareto frontier is obtained by using the dominance relationship among different solutions. 


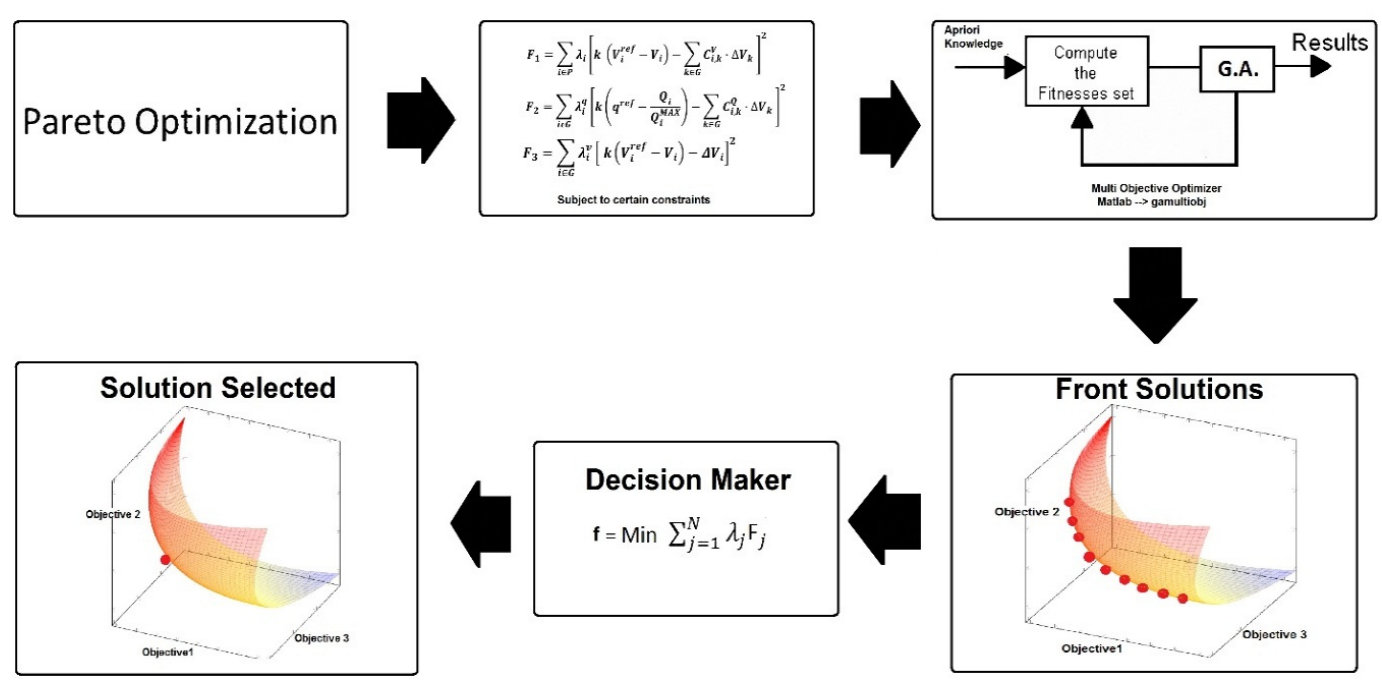

Figure 1. Pareto optimization scheme for a multi-objective problem.

The algorithm needs to choose only one solution to this set of solutions using a new condition decision-maker (DM) [8].

For each set of solutions, the decision-maker (DM) calculates the minimum of the sum of the three objectives; the set of solutions that have the minimum is selected:

$$
f=\operatorname{Min} \sum_{j=1}^{N} \lambda_{j} F_{j}
$$

where:

$f$ is the minimum sum of the objectives of the set of solutions. $N$ is the number of objectives. $\lambda_{j}$ is the weight of the objective $j . F_{j}$ is the objective $j$ of the MO voltage control problem.

\subsection{Fuzzy Logic}

Fuzzy logic is an extension of traditional Boolean relations where the system is not characterized by simple binary values but a range of truths from 0 to 1 . The input and output of the system are "somehow" related [20]. Fuzzy logic is increasingly utilized in distribution networks.

Two of the most important types of fuzzy control are: the Mamdani and Sugeno models. The Mamdani model allows expressing the available prior knowledge of the system, whereas the Sugeno model simplifies the calculations of the output. The Sugeno output can be either linear or constant and the final output is a weighted average of each rule's output; so, its process does not require defuzzification. It works well with optimization and adaptive techniques and has a guaranteed continuity of output surface. Finally, the Sugeno model is well suited to mathematical analysis [25].

In this work, the Sugeno model will be used and its mathematical model has the following form:

$$
\text { If input } 1=x \text {, then the Output is } z=c
$$

In a zero-order model, the output level $z$ is a constant $(\mathrm{a}=0)$. Each output $z_{i}$ of each rule has a weight $w_{i}$ [12]:

$$
w_{i}=\min F_{1}(x)
$$

where $F_{1}(x)$ are the membership functions for input 1 [25]. The average estimate is then given by the equation:

$$
\text { Final output }=\frac{\sum_{i=1}^{N} w_{i} z_{i}}{\sum_{i=1}^{N} w_{i}}
$$


$\mathrm{CVCPF}$ uses fuzzy logic to calculate the optimal reactive power of DG. Figure 2 shows the fuzzy logic reactive power controller. The input signal is the error $(\Delta V)$. This error $(\Delta V)$ is varying over the range $\left[\Delta V_{\min }\right.$, Zero and $\left.\Delta V_{\max }\right]$ where $\Delta V_{\min }=-0.05$ (p.u.) and $\Delta V_{\max }=+0.05$ (p.u.). The output of the fuzzy logic is the variation of the reactive power. The output of the controller is the voltage variation. The PID generates an output based on the difference between the power factor calculated by fuzzy logic and output power factor of the network. The three linguistic labels define voltage: Low, Normal, and High. The input membership (Gaussian) functions are shown in Figure 3.

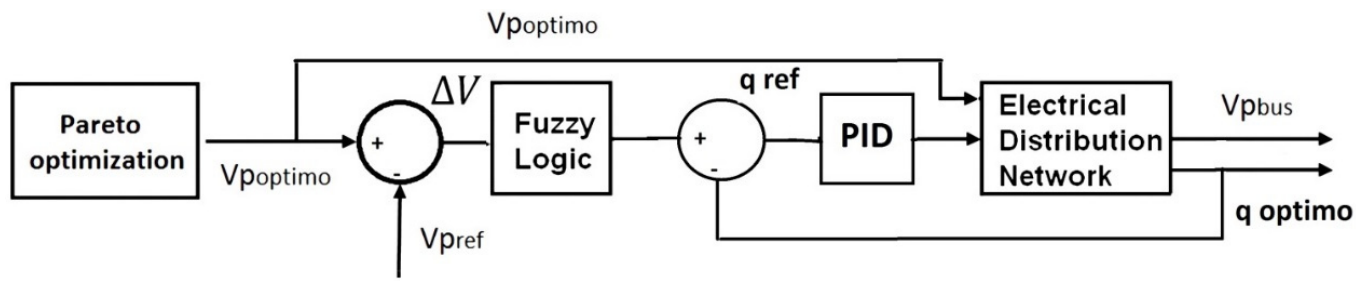

Figure 2. Fuzzy logic reactive power factor controller.

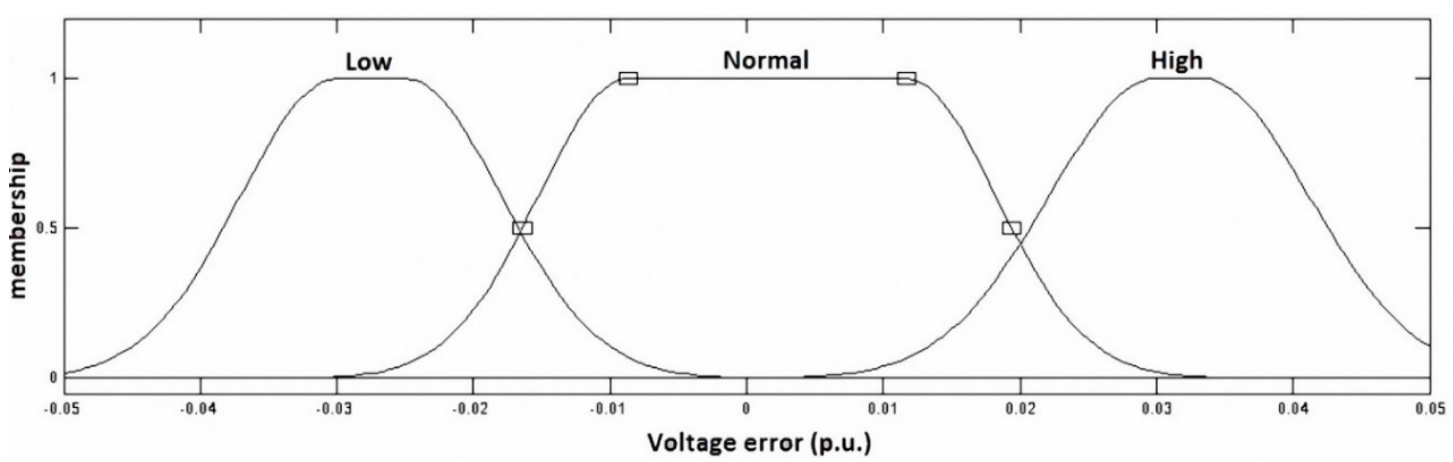

Figure 3. Input fuzzy membership functions.

\subsection{Design of Reactive Power of DG}

In this work, this model is a single-input and single output (SISO) controller (Figure 2). Using relation Equation (8):

$$
\text { If input } 1=\Delta V \text {, then Output is } z=c
$$

The set of fuzzy rules are as follows:

$$
\begin{aligned}
& \text { IF }(\Delta V=\text { Low }) \text { THEN } u_{1}=P F_{\text {min }} \\
& \text { IF }(\Delta V=\text { Normal }) \text { THEN } u_{2}=P F_{\text {nom }} \\
& \text { IF }(\Delta V=\text { High }) \text { THEN } u_{3}=P F_{\max }
\end{aligned}
$$

The advantage of the Sugeno model is that the output can be found using the average estimate formula [25].

$$
P F_{r e f}=\frac{\sum_{i=1}^{3} w_{i} u_{i}}{\sum_{i=1}^{3} w_{i}}
$$

where:

$u_{1}, u_{2}, u_{3}$ are the outputs of the respective fuzzy rules. $w_{i}=\min F_{1}(x)$, when $F_{1}(x)$ is the membership function for input 1. 


\subsection{Solution Algorithm}

The algorithm flow chart is illustrated in Figure 4. The steps followed to solve the MO voltage control problem are as follows:

Step 1. System Data: Define input variables; the algorithm acquires the network values.

Step 2. Analyze and complete the objective functions. The objective functions are calculated from Equations (1) to (3) and the constraints Equations (4) to (6). CVCPF calculates the three weights corresponding to F1, F2, and F3 and finds a set of solutions (Pareto frontier).

Step 3. Decision-maker (DM) calculates the fitness solution.

Step 4. Fuzzy logic

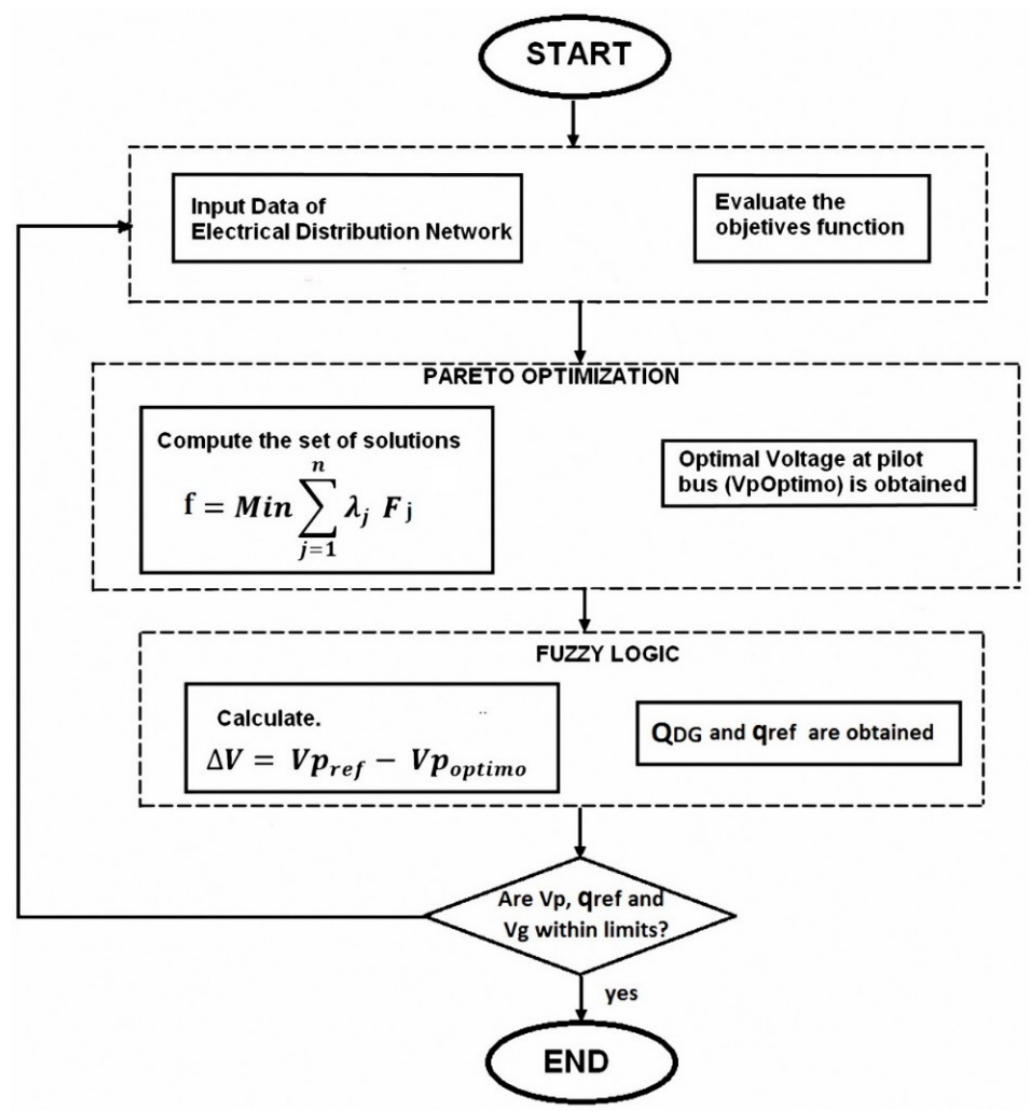

Figure 4. Flow chart of the proposed algorithm.

Figure 4 shows the step 4 . The error $(\Delta V)$ is calculated:

$$
\Delta V=V p_{\text {ref }}-V p_{\text {optimo }}
$$

Determination of the rules: Equation (12) shows the rules.

Determination of the output stage: The final output is computed according to Equation (13). Finally, the reactive power of DG is:

$$
\begin{gathered}
\text { Ang }=\operatorname{acos}(P F) \\
Q_{D G}=(\text { Active power of } D G) \times \tan (\text { Ang })
\end{gathered}
$$

Determination of the optimal reactive power reference: The reactive power is computed using Equation (4):

$$
q^{r e f}=\sum_{\mathrm{i} \in \mathrm{G}} Q_{D G i} / \sum_{\mathrm{i} \in \mathrm{G}} Q_{i}^{M A X}
$$


Finally, the PID removes the error of the power factor.

Step 5. Control: According to the voltage at the pilot bus and the optimal reactive power reference, the control action is calculated on the OLTC and the PF of the DG.

Step 6. With the data from step 5, CVCPF calculates new values for the distribution network using the OpenDSS program [26].

Step 7. If voltage values at the pilot buses, reactive power reference, and voltage at generators are within the limits go to step 8 ; if not, return to step 1.

Step 8. End.

\subsection{Case Study}

The proposed method is tested on IEEE13 Node Test Feeder shown in Figure 5, $4.16 \mathrm{kV}$ distribution network. The technical data of the network is given in [27]. In this work, for Case 1,2, and 3 only a DG with $1.290 \mathrm{~kW}$ connected at the 675 bus is considered [4]. For Case 4, this work uses three DGs.

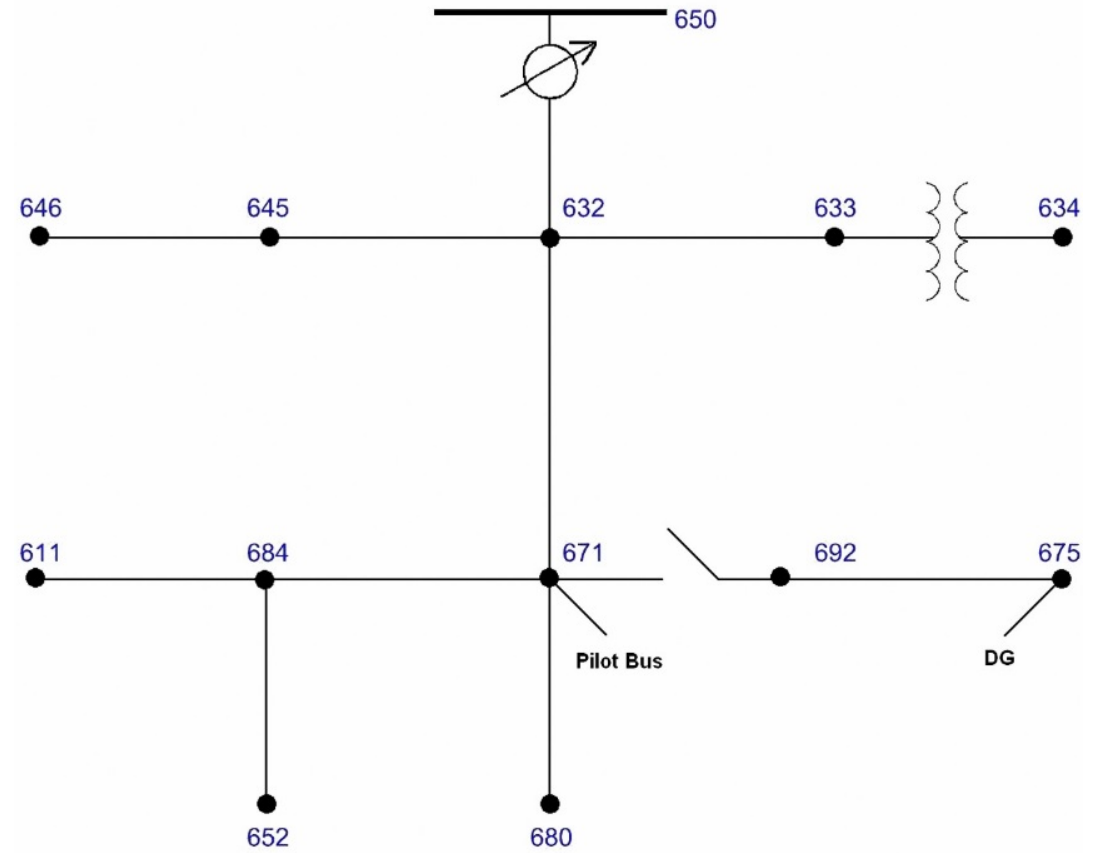

Figure 5. IEEE 13 Node Test Feeder3.5.1. Fixed and Variable Loads.

Table 1 shows the default unbalanced loads values for the network IEEE 13 (fixed values). In the second column of the Table 1, the three basic loads are displayed. (1) Constant Impedance Load Model (Constant Z); (2) Constant Current Load Model (Constant I); and (3) Constant Power Load Model (Constant PQ). In this study, three different cases are analyzed where variable loads are added to the fixed network loads; each case represents the measurements of typical change in consumption of customers in a $48 \mathrm{~h}$ horizon (data provided by Hydro-Québec). Table 2 shows the cable line configuration for an IEEE 13 node test feeder. Figure 6 shows these three cases on the pilot bus in active power (bus 671). 
Table 1. Spot Load Data for IEEE 13.

\begin{tabular}{cccccccc}
\hline Node & Load & $\mathbf{P h - 1}$ & $\mathbf{P h - 1}$ & $\mathbf{P h}-\mathbf{2}$ & $\mathbf{P h - 2}$ & $\mathbf{P h}-\mathbf{3}$ & $\mathbf{P h}-\mathbf{3}$ \\
\hline & Model & $\mathbf{k W}$ & $\mathbf{k V A r}$ & $\mathbf{k W}$ & $\mathbf{k V A r}$ & $\mathbf{k W}$ & $\mathbf{k V A r}$ \\
\hline 634 & Y-PQ & 160 & 110 & 120 & 90 & 120 & 90 \\
645 & Y-PQ & 0 & 0 & 170 & 125 & 0 & 0 \\
646 & D-Z & 0 & 0 & 230 & 132 & 0 & 0 \\
652 & Y-Z & 128 & 86 & 0 & 0 & 0 & 0 \\
671 & D-PQ & 385 & 220 & 385 & 220 & 385 & 220 \\
675 & Y-PQ & 485 & 190 & 68 & 60 & 290 & 212 \\
692 & D-I & 0 & 0 & 0 & 0 & 170 & 151 \\
611 & Y-I & 0 & 0 & 0 & 0 & 170 & 80 \\
\hline & TOTAL & 1158 & 606 & 973 & 627 & 1135 & 753 \\
\hline
\end{tabular}

Table 2. Cable line configuration for IEEE 13 node test feeder.

\begin{tabular}{cccccc}
\hline Node & R (Mile) & X (Mile) & Distance & Config. & X/R Ratio \\
\hline $650-632$ & 0.3465 & 1.0179 & 0.378 & 601 & 2.9376 \\
$632-633$ & 0.7526 & 1.1814 & 0.094 & 602 & 1.5697 \\
$632-645$ & 1.3294 & 1.3471 & 0.094 & 603 & 1.0133 \\
$632-671$ & 0.3465 & 1.0179 & 0.378 & 601 & 2.9376 \\
$645-646$ & 1.3294 & 1.3471 & 0.056 & 603 & 1.0133 \\
$671-684$ & 1.3238 & 1.3569 & 0.056 & 604 & 1.0250 \\
$671-680$ & 0.3465 & 1.0179 & 0.189 & 601 & 2.9376 \\
$692-675$ & 0.7982 & 0.4463 & 0.094 & 606 & 0.5591 \\
$684-611$ & 1.3292 & 1.3475 & 0.056 & 605 & 1.0137 \\
$684-652$ & 1.3425 & 0.5124 & 0.151 & 607 & 0.3816 \\
$671-692$ & & & & Switch & \\
$633-634$ & $1.10 \%$ & $2 \%$ & & XFM-1 & \\
\hline
\end{tabular}

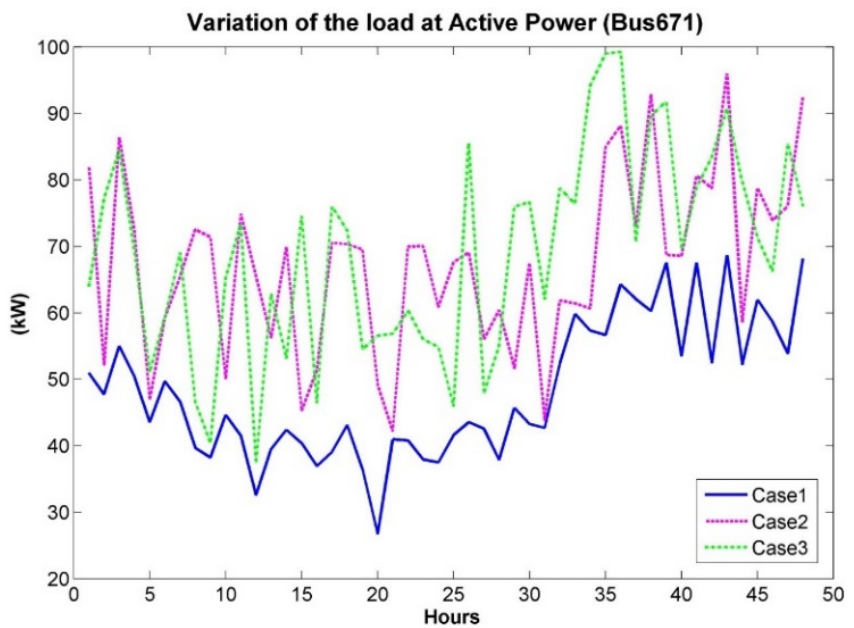

Figure 6. Variation of the load in $\mathrm{kW}$ at Bus 671.

In Figure 6 and in the Table 3, we can see the maximum load variations. Case 1 is 16.27 and $16.49 \mathrm{~kW}$ at hours 42 to 43 and 43 to 44 , respectively; Case 2 is 34.28 and $37.38 \mathrm{~kW}$ at hours 2 to 3 and 43 to 44 , respectively; and Case 3 is 39.66 and $37.73 \mathrm{~kW}$ at hours 25 to 26 and 26 to 27, respectively. 
Table 3. Maximum load variations in Case 1, 2 and 3.

\begin{tabular}{|c|c|c|c|c|c|c|c|c|}
\hline \multicolumn{3}{|c|}{ Case $1(\mathbf{k W})$} & \multicolumn{3}{|c|}{ Case $2(\mathrm{~kW})$} & \multicolumn{3}{|c|}{ Case $3(\mathrm{~kW})$} \\
\hline Hour & Bus 671 & Variation & Hour & Bus 671 & Variation & Hour & Bus 671 & Variation \\
\hline 43 & 68.69 & 16.27 & 3 & 86.38 & 34.28 & 26 & 85.59 & 39.66 \\
\hline 44 & 52.20 & 16.49 & 44 & 58.62 & 37.38 & 27 & 47.86 & 37.73 \\
\hline
\end{tabular}

\section{Simulation Results}

The proposed method (CVCPF) is compared with two other methods (OLTC and OCVC).

In the method OLTC, the only equipment used for the voltage control is the OLTC. This is the typical case of a distribution network nowadays. The connection of DG and the variable load will fundamentally alter the feeder voltage profile then the OLTC performs control voltage. The reactive power injected from the DG is zero in this method; furthermore, the DG does not participate in the regulation of the voltage.

Optimal Coordinated Voltage Control (OCVC) proposes a solution for the MO voltage control problem using only Pareto optimization. This method proposes a balanced participation in the reactive power of DG connected to the distribution network. In OCVC, the weighting factors vary dynamically depending on: (1) the value of the voltage at the pilot bus, (2) the value of the voltage at the bus generator, and (3) the value of the reactive power available [10].

The difference between CVCPF and OCVC is that CVCPF uses two techniques to calculate the optimum values. OCVC uses only Pareto to get the optimum values whereas CVCPF uses Pareto and fuzzy logic. To calculate the reactive power given by DG, CVCPF uses fuzzy logic according to the optimum values given by Pareto.

The effect of reactive power of DG on the voltage profile and the variable load in the network is shown in Figures 7-9. In all three cases, the reactive power input of CVCPF and OCVC are almost equal. The difference is the voltage variation; in the CVCPF method it is lower than in the other methods (Table 3 ).
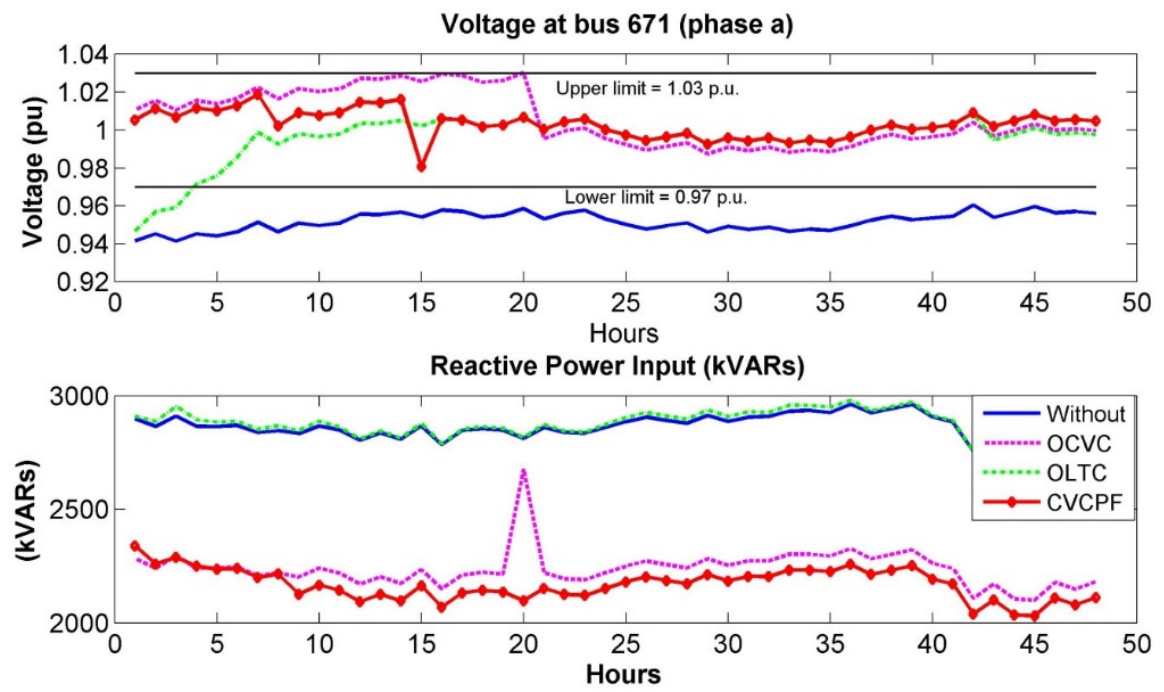

Figure 7. Voltage at bus 671 (phase a) with respect to reactive power input. Case 1. 

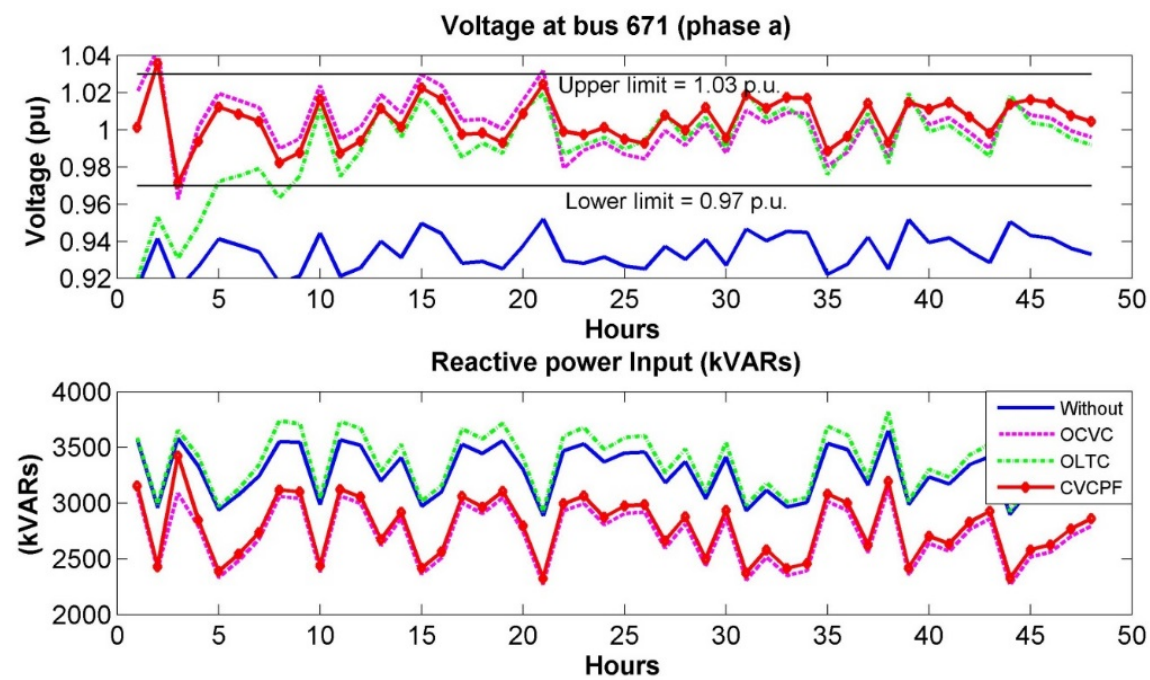

Figure 8. Voltage at bus 671 (phase a) with respect to reactive power input. Case 2.

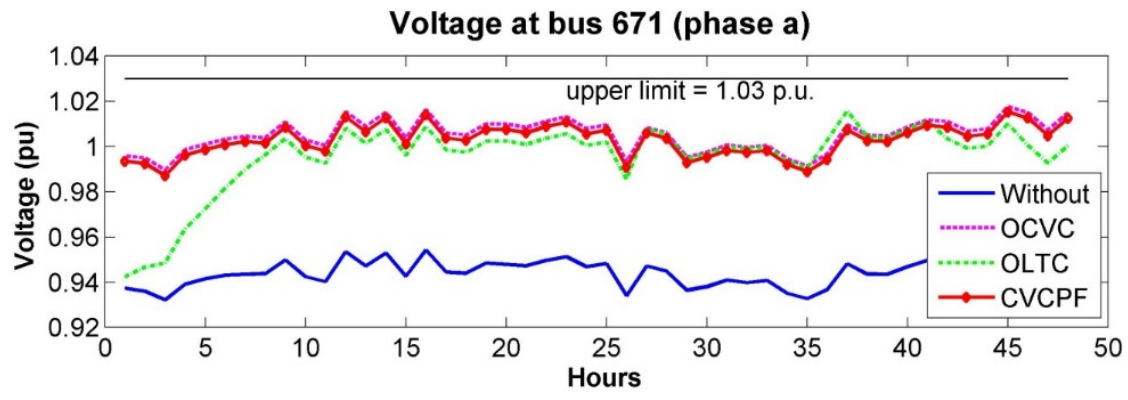

Reactive Power Input (kVARs)

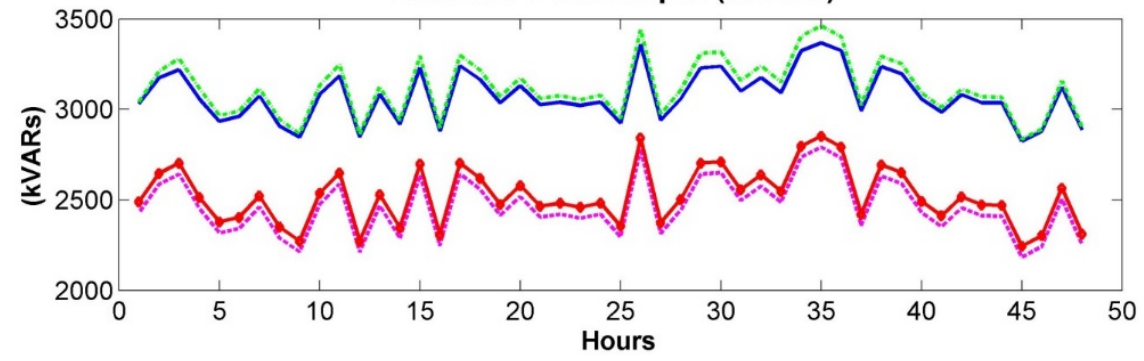

Figure 9. Voltage at bus 671 (phase a) with respect to reactive power input. Case 3.

For the case study, the constraints of Equations (4) and (5) will be:

$$
\begin{gathered}
\left|Q_{i}\right| \leqslant D G(k W) \times( \pm 0.91) \\
V_{i} \in[0.97 ; 1.03] \text { for } i \in P \cup G
\end{gathered}
$$

In the method "without", the network does not perform any voltage control. The DG and variable loads cause voltage variations.

Case 1:

In Figure 7, we can see that when the voltage reaches the upper limit allowed, the Objective 1 of the MO voltage control problem is the priority (Equation (1)). The voltage at hour 20 (OCVC line) reaches the maximum allowed value; OCVC maintains the voltage close to the reference value. Objective 2 of the MO voltage control problem is not the priority (Equation (2)), so the reactive power of the DG decreases and the reactive power input increases. 
From hour 21, the profile voltages are similar. However, in CVCPF the voltage is close to one (1 p.u.). Reactive power input is similar in these two methods.

In the hours 43 and 44 (maximum load variations), the variation of voltage in reactive power is similar in the CVCPF and OCVC methods.

Case 2:

At hours 3 and 44 (maximum load variations) of Figure 8 and Table 4 , the voltage variation in CVCPF is smaller than in the other methods. At hour 3, OLTC has a lower variation than CVCPF but the voltage on the bus 671 is not within the limits (Figure 8). In the hours 3, 22, 39, and 44, we can see that each time that the CVCPF line crosses the OCVC line; the voltage variation in CVCPF is smaller than the other methods. Additionally, at this time, the reactive power input between CVCPF and OCVC is almost similar. So, CVCPF used DG reactive power to reduce the voltage variation.

Table 4. Voltage variation. Case 2. CVCPF, OCVC, and OLTC methods.

\begin{tabular}{ccccc}
\hline \multirow{5}{*}{ Case 2 } & \multicolumn{3}{c}{ Variation (V p.u.) } \\
\cline { 3 - 5 } & Hour & CVCPF & OCVC & OLTC \\
\cline { 3 - 5 } Maximum load variation & 3 & 0.065 & 0.081 & 0.033 \\
& 44 & 0.016 & 0.026 & 0.033 \\
\hline \multirow{2}{*}{ Line crosses } & 3 & 0.065 & 0.081 & 0.033 \\
& 22 & 0.026 & 0.053 & 0.033 \\
& 39 & 0.021 & 0.032 & 0.038 \\
OCVC variation is & 44 & 0.016 & 0.026 & 0.033 \\
\hline higher than $0.025 \mathrm{~V}$ & 3 & 0.065 & 0.081 & 0.033 \\
& 4 & 0.023 & 0.039 & 0.024 \\
& 10 & 0.028 & 0.029 & 0.036 \\
& 11 & 0.028 & 0.029 & 0.036 \\
& 22 & 0.026 & 0.053 & 0.033 \\
& 35 & 0.028 & 0.029 & 0.029 \\
& 39 & 0.021 & 0.032 & 0.038 \\
& 44 & 0.016 & 0.026 & 0.033 \\
\hline
\end{tabular}

When the voltage variation on the method OCVC is higher than 0.025 p.u. (Table 4), the voltage in CVCPF is lower. This can be observed at the hours 3, 4, 10, 11, 22, 35, 39, and 44. At these hours, there is a small difference between the reactive power input of CVCPF and OCVC. Fuzzy logic is better suited to voltage changes caused by the variation of the load. Therefore, fuzzy logic achieves a more efficient management of reactive power.

Case 3:

At hours 26 and 27 (maximum load variations) of Figure 9, the voltage variation in CVCPF is similar than in the other methods. In all the time, voltage variations in CVCPF and OCVC have not exceeded the value of 0.025 p.u. Similarly, the reactive power input for CVCPF and OCVC are similar.

The losses of active and reactive power for CVCPF and OCVC are always lower than other proposed methods (Figure 10). 

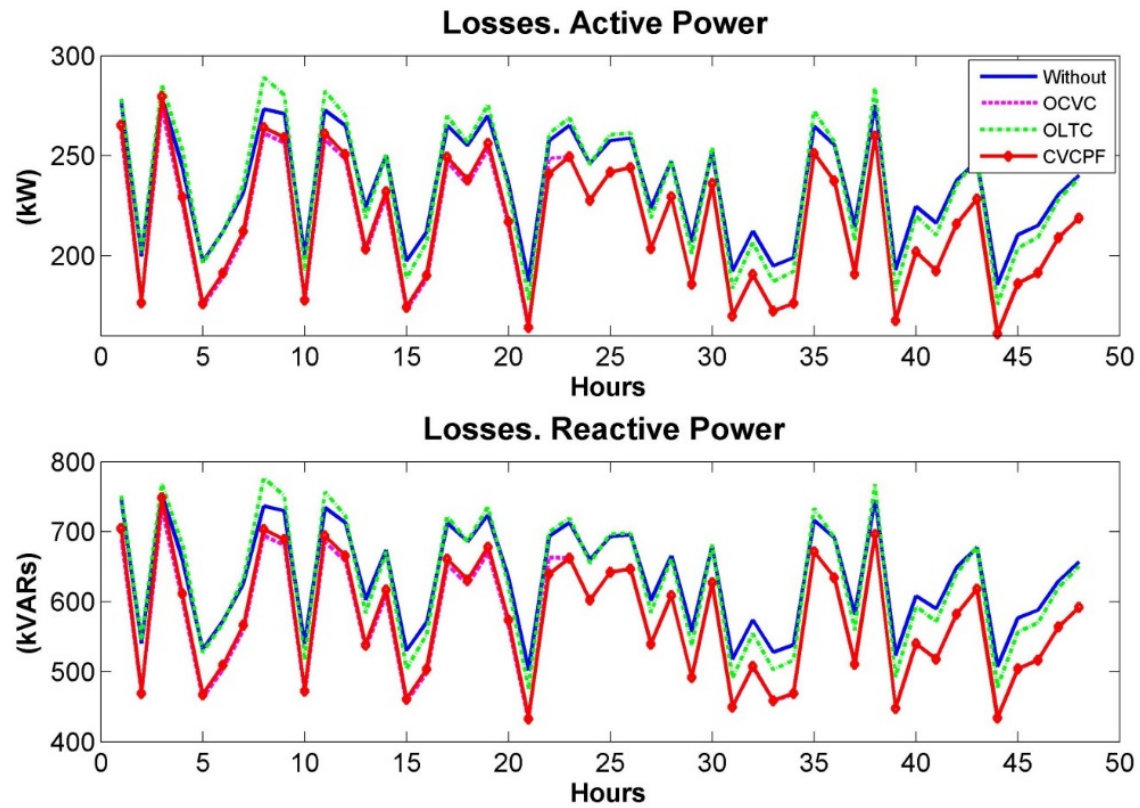

Figure 10. Losses. Active and reactive power for Case 2.

Figure 11 shows the reactive power delivered by the DG for Case 3 using CVCPF and OCVC methods. The reactive power varies according to the needs of the network. Then, the reactive power of the DG helps the distribution network to maintain a stable voltage and reduce loss.

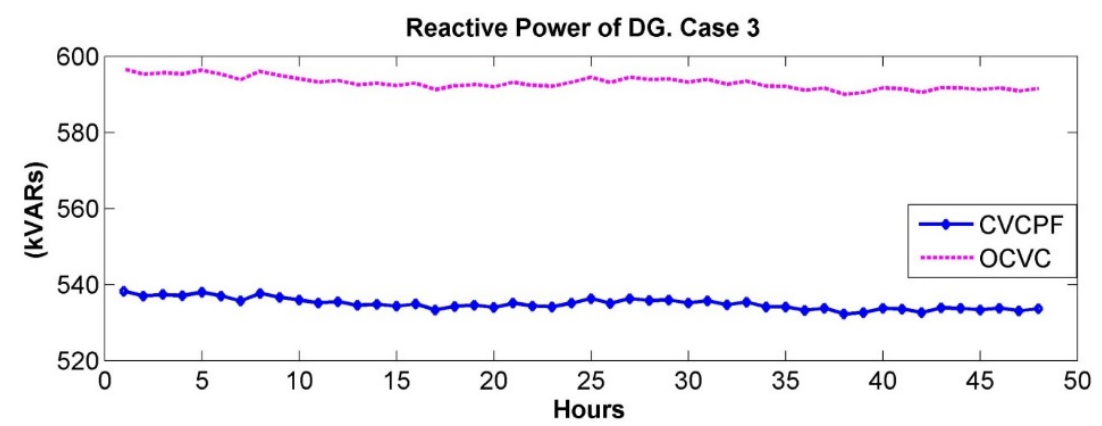

Figure 11. Reactive power generated by the DG for Case 3 with CVCPF and OCVC methods.

For the simulation, the OpenDSS and Matlab programs are used. We have used OpenDSS for unbalanced load flow. The method uses an OpenDSS server to communicate with Matlab; thus, OpenDSS data and Matlab can work together.

Case 4.

The IEEE 13 Node Test Feeder has three DGs. The DG1 is located on the bus 675 and has a capacity of $360 \mathrm{~kW}$. The DG2 is located on the bus 671 and has a capacity of $630 \mathrm{~kW}$. Finally, The DG3 is located on the bus 632 and has a capacity of $300 \mathrm{~kW}$ [28]. Variable load 1 is used in this case.

The Figure 12 shows that the voltage at the pilot bus is always within the limits. However, in CVCPF the voltage variation is less. 

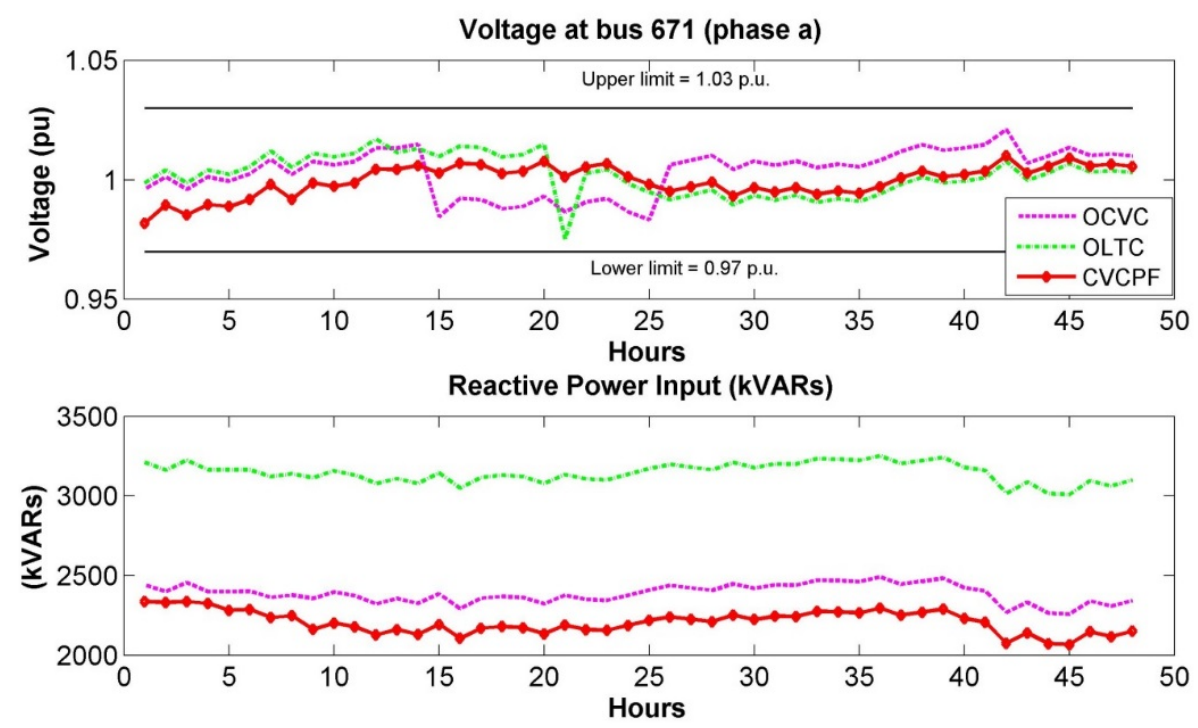

Figure 12. Voltage at pilot bus with respect to reactive power input. Case 4.

\section{Conclusions}

A new algorithm, called CVCPF, for resolving the MO voltage control problem in distribution networks is presented. The three objectives considered in this paper are: voltage at pilot bus, management of the reactive power and voltage in generators. CVCPF uses a combination of optimization techniques (Pareto optimization and fuzzy logic) to find the optimal values for the $\mathrm{MO}$ voltage control problem.

The performance of the CVCPF is evaluated on an IEEE 13 node test feeder. Variables and unbalanced loads are used, based on real consumption data, over a time window of $48 \mathrm{~h}$. Three such profiles are used in the study, varying in the amount of the load. The results are compared with those obtained from the methods OCVC and OLTC as well as from the case of no voltage control.

This work demonstrates that CVCPF reduces the voltage variation more than the other methods.

This work shows also that optimal integration of the DGs in the distribution network helps to maintain stable voltage and to reduce loss.

CVCPF includes the use of decision-maker; in this study the fitness solution was used but various options are possible. The use of CVCPF could be advantageous with respect to the development of a flexible system for network operators, by applying different settings at the decision stage, according to specific circumstances. Further research is needed on this topic.

Acknowledgments: Thanks to Ecole de technologie supérieure and Hydro Québec for the support given in this work.

Author Contributions: José Raúl Castro is the author of this work under the direction of Maarouf Saad. All co-authors made suggestions for the research and contributed to the writing of the final research paper.

Conflicts of Interest: The authors declare no conflict of interest.

\section{References}

1. Habibi, A.; Nayeripour, M.; Aghaei, J. Secure multi-objective distributed generation planning in distribution network. In Proceedings of the 2013 21st Iranian Conference on Electrical Engineering (ICEE), Mashhad, Iran, 14-16 May 2013; pp. 1-6.

2. De Oliveira-de Jesus, P.M.; de Leao, M.T.P.; Khodr, H.M. Remuneration of distribution networks using a fuzzy multicriteria planning algorithm. In Proceedings of the 2006 International Conference on Probabilistic Methods Applied to Power Systems (PMAPS 2006), Stockholm, Sweden, 11-15 June 2006; pp. 1-7. 
3. Ahmidi, A.; Guillaud, X.; Besanger, Y.; Blanc, R. A multilevel approach for optimal participating of wind farms at reactive power balancing in transmission power system. IEEE Syst. J. 2012, 6, 260-269. [CrossRef]

4. Anwar, A.; Pota, H.R. Loss reduction of power distribution network using optimum size and location of distributed generation. In Proceedings of the 2011 21st Australasian Universities Power Engineering Conference (AUPEC), Brisbane, Australia, 25-28 September 2011; pp. 1-6.

5. Queiroz, L.M.O.; Lyra, C. Adaptive hybrid genetic algorithm for technical loss reduction in distribution networks under variable demands. IEEE Trans. Power Syst. 2009, 24, 445-453. [CrossRef]

6. Zidan, A.; El-Saadany, E.F. Network reconfiguration in balanced distribution systems with variable load demand and variable renewable resources generation. In Proceedings of the 2012 IEEE Power and Energy Society General Meeting, San Diego, CA, USA, 22-26 July 2012; pp. 1-8.

7. Lopez, E.; Opazo, H.; Garcia, L.; Bastard, P. Online reconfiguration considering variability demand: Applications to real networks. IEEE Trans. Power Syst. 2004, 19, 549-553. [CrossRef]

8. Dehghani-Arani, A.; Maddahi, R. Introduction a multi-objective function in unbalanced and unsymmetrical distribution networks for optimal placement and sizing of distributed generation units using NSGA-II. In Proceedings of the 2013 18th Conference on Electrical Power Distribution Networks (EPDC), Kermanshah, Iran, 30 April-1 May 2013; pp. 1-9.

9. Hong, Y.Y.; Lai, Y.M.; Chang, Y.R.; Lee, Y.D.; Liu, P.W. Optimizing capacities of distributed generation and energy storage in a small autonomous power system considering uncertainty in renewables. Energies 2015, 8, 2473-2492. [CrossRef]

10. Richardot, O.; Viciu, A.; Besanger, Y.; HadjSaid, N.; Kieny, C. Coordinated voltage control in distribution networks using distributed generation. In Proceedings of the 2005/2006 IEEE PES Transmission and Distribution Conference and Exhibition, Dallas, TX, USA, 21-24 May 2006; pp. 1196-1201.

11. Knowles, J.; Corne, D. The pareto archived evolution strategy: A new baseline algorithm for pareto multiobjective optimisation. In Proceedings of the 1999 Congress on Evolutionary Computation, Washington, DC, USA, 6-9 July 1999; Volume 101, p. 105.

12. Soroudi, A.; Caire, R.; Hadjsaid, N.; Ehsan, M. Probabilistic dynamic multi-objective model for renewable and non-renewable distributed generation planning. IET Gener. Transm. Distrib. 2011, 5, 1173-1182. [CrossRef]

13. Ngatchou, P.; Anahita, Z.; El-Sharkawi, M.A. Pareto multi objective optimization. In Proceedings of the 13th International Conference on Intelligent Systems Application to Power Systems, Arlington, VA, USA, 6-10 November 2005; pp. 84-91.

14. Lin, X.; Yao, J.; Mao, T.; Gong, L. Inductive reactive power compensation optimization for $10 \mathrm{kV}$ distribution network with distributed generations. In Proceedings of the 2013 Chinese Automation Congress (CAC), Changsha, China, 7-8 November 2013; pp. 87-91.

15. Deb, K.; Pratap, A.; Agarwal, S.; Meyarivan, T. A fast and elitist multiobjective genetic algorithm: NSGA-II. IEEE Trans. Evol. Comput. 2002, 6, 182-197. [CrossRef]

16. Barin, A.; Canha, L.N.; Machado, R.Q.; da Rosa Abaide, A.; Maziero, E.; Arend, G. Analysis of the impacts of distributed generation sources considering the appropriate choice of parameters in a multi-objective approach for distribution system planning. In Proceedings of the 5th International Conference on European Electricity Market, Lisboa, Portugal, 28-30 May 2008; pp. 1-6.

17. Loetamonphong, J.; Fang, S.C.; Young, R.E. Multi-objective optimization problems with fuzzy relation equation constraints. Fuzzy Sets Syst. 2002, 127, 141-164. [CrossRef]

18. Gao, Y.; Liu, J.; Yang, J.; Liang, H.; Zhang, J. Multi-objective planning of multi-type distributed generation considering timing characteristics and environmental benefits. Energies 2014, 7, 6242-6257. [CrossRef]

19. Gaonkar, J.D.N.; Pillai, G.N. Fuzzy logic based coordinated voltage regulation method for distribution system with multiple synchronous generators. In Proceedings of the 2010 IEEE PES Transmission and Distribution Conference and Exposition, New Orleans, LA, USA, 19-22 April 2010; pp. 1-5.

20. Ghatee, M.; Hashemi, S.M. Application of fuzzy minimum cost flow problems to network design under uncertainty. Fuzzy Sets Syst. 2009, 160, 3263-3289. [CrossRef]

21. Kiprakis, A.E.; Wallace, A.R. Maximising energy capture from distributed generators in weak networks. IEE Proc. Gener. Transm. Distrib. 2004, 151, 611-618. [CrossRef]

22. Maciel, R.S.; Padilha-Feltrin, A. Distributed generation impact evaluation using a multi-objective tabu search. In Proceedings of the 15th International Conference on Intelligent System Applications to Power Systems, Curitiba, Brazil, 8-12 November 2009; pp. 1-5. 
23. Viawan, F.A.; Karlsson, D. Coordinated voltage and reactive power control in the presence of distributed generation. In Proceedings of the 2008 IEEE Power and Energy Society General Meeting-Conversion and Delivery of Electrical Energy in the 21st Century, Pittsburgh, PA, USA, 20-24 July 2008; pp. 1-6.

24. Masters, C.L. Voltage rise: The big issue when connecting embedded generation to long $11 \mathrm{kv}$ overhead lines. Power Eng. J. 2002, 16, 5-12. [CrossRef]

25. Takagi, T.; Sugeno, M. Fuzzy identification of systems and its applications to modeling and control. IEEE Trans. Syst. Man Cybern. 1985, SMC-15, 116-132. [CrossRef]

26. Opendss Manual and Reference Guide. Available online: http://sourceforge.net/projects/electricdss (accessed on 18 May 2015).

27. Kersting, W.H. Radial distribution test feeders. In Proceedings of the 2001 IEEE Power Engineering Society Winter Meeting, Columbus, OH, USA, 28 January-1 February 2001; Volume 902, pp. 908-912.

28. Khushalani, S.; Schulz, N. Unbalanced distribution power flow with distributed generation. In Proceedings of the 2005/2006 IEEE PES Transmission and Distribution Conference and Exhibition, Dallas, TX, USA, 21-24 May 2006; pp. 301-306.

(C) 2016 by the authors; licensee MDPI, Basel, Switzerland. This article is an open access article distributed under the terms and conditions of the Creative Commons by Attribution (CC-BY) license (http://creativecommons.org/licenses/by/4.0/). 